\title{
Correlations between mutant huntingtin aggregates and behavioral changes
}

in $\mathrm{R} 6 / 1$ mice.

Magali CABANAS $^{\mathrm{a}, \mathrm{b}}$, Marion PIQUEMAL ${ }^{\mathrm{a}, \mathrm{b}}$, Cristiana PISTONO ${ }^{\mathrm{a}, \mathrm{b}}$, Syndelle ARNAUD ${ }^{\mathrm{a}, \mathrm{b}}$, Divyangana RAKESH ${ }^{a, b}$, Elodie Poinama ${ }^{a, b}$, Jean-Louis Guillou ${ }^{a, b}$, Maurice GARRET ${ }^{a, b}$, Yoon H. $\mathrm{CHO}^{\mathrm{a}, \mathrm{b}, \mathrm{c}}$

a. Institute of Cognitive and Integrative Neuroscience of Aquitaine, CNRS UMR 5287

b. Institute of Cognitive and Integrative Neuroscience of Aquitaine, University of Bordeaux

Running title: mutant huntingtin and behavioral deficits in R6/1 mice

c. Corresponding author: Yoon H. CHO, Ph.D. Institute of Cognitive and Integrative Neuroscience of Aquitaine, CNRS UMR 5287, Allee Geoffroy St Hilaire, CS 50023, 33615 Pessac Cedex, France, Phone: +33 (0) 5400087 46, Fax: +33 (0) 400087 42, Email: yoon.cho@u-bordeaux.fr 


\section{Abstract}

BACKGROUND: Huntington's disease (HD) is a neurodegenerative disorder caused by the expansion of the trinucleotide CAG in the HD gene. While the presence of nuclear aggregates of mutant huntingtin ( $\mathrm{mHtt}$ ) in neurons is a hallmark of $\mathrm{HD}$, the reason behind its toxicity remains elusive.

OBJECTIVE: The present study was conducted to assess a correlation between the number of $\mathrm{mHtt}$ aggregates and the severity of HD symptoms in R6/1 mice.

METHODS: We investigated correlations between behavioral deficits and the level of nuclear $\mathrm{mHtt}$ aggregates in different neuroanatomical regions in 3-month-old R6/1 mice, the age at which a large variability of symptom severity between animals has been observed.

RESULTS: R6/1 mice were deficient in instinctive and anxiety related behaviors as well as long-term memory capabilities. Significant differences were also found between the sexes; female transgenic mice displayed less severe deficits than males. While the level of $\mathrm{mHtt}$ aggregates was correlated with the severity of HD phenotypes in most regions of interest, an opposite relationship also was found for some other regions examined.

CONCLUSIONS: The obtained results suggest harmful and region specific roles of $\mathrm{mHtt}$ aggregates in HD symptoms.

\section{Keywords}

Mutant huntingtin aggregates, motor activity, short-term and long-term memory, spatial memory, anxiety, instinctive behavior, R6/1 mice 


\section{Introduction}

Huntington's disease (HD) is caused by the mutation of a single gene that encodes for huntingtin (Htt) protein. The mutated HD gene, with the expansion of the trinucleotide CAG, codes for a mutated protein $(\mathrm{mHtt})$ that tends to aggregate in the nucleus of neural cells during the course of the disease (1). While mHtt nuclear aggregates remain an uncontestable hallmark of HD pathology $(2,3)$, their exact role in the development of clinical symptoms and neurodegeneration remains controversial. More precisely, early observations of the co-occurrence of these nuclear aggregates and cell death contributed to the hypothesis of the toxic gain of function of $\mathrm{mHtt}(4,5)$. Further investigations, however, demonstrated that there exists no correlation between the number of aggregates and neurodegeneration, as brain regions heavily invaded by aggregates are not necessarily the ones that degenerate as first in HD. In addition, the massive amount of $\mathrm{mHtt}$ aggregates in the nucleus was not sufficient for neurodegeneration or cell loss to occur (6). Other studies described the inclusions as incidental or even neuroprotective; according to these studies, the inclusions may reflect a cellular coping process against harmful events caused by the HD mutation (7-10).

To obtain further insight into the potential functional contribution of nuclear $\mathrm{mHtt}$ microaggregates to HD associated symptoms and symptom severity, we performed a correlative study involving a multidisciplinary approach. A large cohort of male and female R6/1 mice were submitted to a battery of behavioral tests and then promptly to a post-mortem analysis for $\mathrm{mHtt}$ microaggregates in different cortico-striatal and limbic circuits. $R 6 / 1$ is a transgenic line, with IT15 (human huntingtin gene) used as promoter. The distribution of mHtt aggregates in the R6/1 model has been already described in the literature (Naver et al.; Rattray I et al 2013 for ex.). The R6/1 mice were tested at the age of 3 months. This age corresponds to a turning point in the R6/1 mouse line where some mice develop apparent behavioral deficits while others remain relatively symptom free. While the level of $\mathrm{mHtt}$ aggregates was correlated with the severity of HD phenotypes in most regions of 
interest, an opposite relationship was also found for some other areas examined. These results suggest harmful and region specific roles of $\mathrm{mHtt}$ aggregates in HD symptoms.

\section{Materials and Methods}

\section{Ethics Statement}

Experimental procedures reported here were approved by the local Institutional Animal Care and Use Committee (Comité d'Ethique pour l'Expérimentation Animale Bordeaux), and followed European Communities Council Directive of 24 November 1986 (86/609/ EEC).

\section{Animals}

Subjects were male and female R6/1 (B6.Cg-Tg (HDexon1) 61Gpb/J, Stock number: 006471, Jackson Laboratory, Main Harbor, NY, USA) and age-matched wild-type (WT) littermates. The R6/1 transgenic mouse lines express a transgene encoding the 5' end of human HTT (Mangriani, 996). While the CAG repeat size was not determined for the mice used in this experiment, the number in the breeders was 142.188 ( \pm 1.195$)$. Mice came from 16 different litters including 2 to 7 littermates of both sexes. PCR was performed on tail biopsy at weaning, and reconfirmed upon sacrifice for verification. 3-4 month old NMRI female mice (Janvier, Le Genest- Saint-Isle, France) were used as stimulus animals in the social test. All animals were housed in unisexual groups (3-5 per cage) in polycarbonate standard

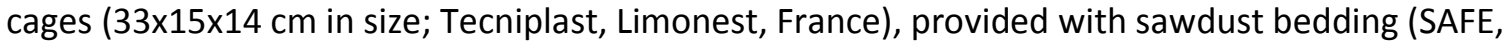
Augy, France), and a stainless steel wire lid. Food chow (SAFE, Augy, France) and water were provided ad libitum unless otherwise mentioned. The animals were maintained in separate male and female colony rooms with temperature $\left(22^{\circ} \mathrm{C}\right)$ and humidity $(50 \%)$ being controlled. Mean ages were $85.80 \pm 1.76$ days for WT mice ( $n=24 ; 15$ males and 9 females) and $87.67 \pm 3.00$ days for Tg mice ( $n=38 ; 21$ males and 17 females) at the beginning of the experiment.

\section{Experimental protocol}


All mice were handled for 5 days and submitted to the same sequence of behavioral testing that lasted 8 consecutive days. They were tested in the elevated plus-maze, and their body weight was recorded on day 1 . On day 2 , they received 3 trials on the rotarod with 1 hour inter-trial interval. On day 3, the mice underwent Y-maze testing followed by social interaction with at least a 1 hour interval between tests. Clasping and grip strength were measured on day 4 followed by one trial of habituation in the Barnes maze. Mice then underwent a one day acquisition period in the Barnes maze ( 6 trials) on day 5 , which was followed by 4 trials of retention testing 24 hours later (day 6 ). On the evening of the $6^{\text {th }}$ day, mice were provided with nesting material, and their nest scores were recorded the following morning (day 7). On day 8 , mice were weighed again and then sacrificed.

During the entire behavioral testing, the mice were transferred to individual cages and testing rooms 1 hour prior to behavioral testing, and returned to their home cages following the behavioral experiments on all days except days 7 and 8 .

\section{Clasping, inverted grid test and rotarod}

For the tail clasping test, mice were suspended by the tail for $10 \mathrm{~s}$; if the mouse acquired a locked body position the result was scored as positive. For grip strength, each mouse was placed on a standard cage lid with metal grid. Prior to rotating the lid $180^{\circ}$ through the horizontal plane the lid was shaken gently, inducing the mice to grip the grid. Then, the latency to fall, up until a maximum of $60 \mathrm{~s}$, was recorded. Motor coordination and balance of mice were assessed in the standard accelerating rotarod apparatus (Immetronic, Pessac, France), with the rotating speed increasing from 4 to 40 rounds per min during the 3 -min trial. Mice were given 3 trials with at least $30 \mathrm{~min}$ to 1 hour inter-trial interval. The latency to fall from the rod, up until a maximum of $3 \mathrm{~min}$, was recorded.

\section{Direct social interaction with an adult female, and nest building behavior}


Subjects were put in contact with an unfamiliar stimulus mouse (a 12-week old NMRI female) and video-recorded in their individual holding cage during a 3 min-session. An observer unaware of the genotype of the animals scored the time spent performing social affiliative (sniffing the head, snout and anogenital part of the body of the partner) and nonsocial (rearing, selfgrooming and digging) behaviors offline. During the encounter, aggressive behaviors and postures, such as attack as well as tail rattling were also identified. Nesting behavior was observed by providing a Neslet $(5 \mathrm{~cm}$ square compressed cotton, Bellmore, NY) in the cage of individually housed mice approximately $1-2 \mathrm{~h}$ prior to the onset of the dark (active) phase. The nest quality was assessed the next morning on a rating scale of 1-5 (1: cotton untouched, 5: perfect tridimensional nest) according to the standard scoring technique(11)

\section{Anxiety in elevated plus maze and spatial recognition memory in Y-maze}

The plus maze was comprised of four arms ( $5 \mathrm{~cm}$ wide, $30 \mathrm{~cm}$ long) made of grey PVC. The maze was placed in a room and elevated $1 \mathrm{~m}$ above the floor. Two opposing arms were closed by $24 \mathrm{~cm}$ high walls, while the other two remained open. A camera was placed above the maze for video recording and tracking of the mice for their locomotion by Ethovision 9 (Noldus Technology, Wageningen, The Netherlands). Mice were individually released from the center of the maze and could move freely around the maze for $8 \mathrm{~min}$. The number of visits as well as total time spent in open versus closed arms were collected and expressed as \% in open arms.

A grey plastic Y-maze was used for short-term spatial recognition memory testing. The maze was placed on an $80 \mathrm{~cm}$ high table and located in a room containing extra-maze visual cues. The three arms (42 cm long, $8 \mathrm{~cm}$ wide, $15 \mathrm{~cm}$ high) of the Y-maze were identical in appearance, and spaced at $120^{\circ}$ from each other. Mice were assigned two arms (start and familiar arms) to which they were exposed during the first phase of the test (sample phase). The remaining third arm, blocked by a transparent door placed at the entrance, constituted the novel arm during the second phase (test 
phase). Allocation of arms (start, familiar, and novel) was counterbalanced within each experimental group. During the sample phase, mice were placed at the end of the designated start arm and allowed to explore freely both the start and the other unblocked (familiar) arm for 5 min. Mice were then removed from the maze and returned to a waiting cage for $10 \mathrm{~min}$ for a retention interval, before the test phase began. In the interval between the sample and the test phase, the apparatus was cleansed with alcohol and water to remove odor residue. During the test phase, the door blocking the novel arm was removed. Mice were placed at the end of the same start arm and allowed to explore the entire maze for $2 \mathrm{~min}$. Timing of the $2 \mathrm{~min}$ test phase period was initiated only once the mouse had left the start arm. Time spent in each arm of the maze during both phases of the experiment was extracted. A spatial recognition memory index was calculated as the time spent in the novel arm divided by the time spent in all three arms $\times 100$.

\section{Learning and memory in a Barnes maze competition task}

The Barnes maze consisted of a circular white surface $(110 \mathrm{~cm}$ diameter) with 18 holes $(5 \mathrm{~cm}$ diameter) around its circumference. Extra-maze visual cues (e.g. wall decoration, furniture in the room) provided animals with spatial references. The room was illuminated excessively ( 270 lux) and a wind blowing from a fan was used to provide a further aversive environment to the rodent. The task was an adaptation of previously published procedures using competition between hippocampusdependent spatial learning and striatum-dependent cued learning in the water maze (12-14) or in the Barnes maze (15). For acquisition, one arbitrary chosen hole gave access to a shelter, whose location could be determined using the layout of extra-maze cues in the room (spatial strategy). In addition to the extra-maze cues, a salient local cue (a plastic water bottle of $50 \mathrm{cl}$ ) was positioned right next to the hole associated with the shelter so that the mice could also be guided by the intra-maze cue (cued strategy). 
The task took place over three consecutive days; a single session of habituation on the first day, 6 acquisition trials the next day and 3 retention trials on the final $3^{\text {rd }}$ day. The mice were released from the center of the arena and were tracked until they reached the hole leading to the shelter. The 3 min cut-off period was used after which mice were gently guided to the safe hole for their entering. The mice remained in the safe hole for $30 \mathrm{sec}$ before being removed and replaced in their holding cages. The inter-trial interval was approximately 10 minutes. Latencies and distances travelled to reach the safe hole as well as moving speeds were collected for each trial. During the retention testing phase, mice were given 3 consecutive trials with the local cue being displaced and associated to a second shelter, placed under the hole, positioned at $180^{\circ}$ relative to the safe hole in acquisition. The remaining procedure was identical to the acquisition trials. If a mouse entered the shelter located at the previous learned location, the response was coded as a "spatial response", and if a mouse entered the new shelter associated with the displaced local cue, it was coded as a "cue response". In addition to the previous measures, the number of unsafe hole visits (errors) were recorded during retention testing.

\section{Immunofluorescence}

The mice were deeply anesthetized with an overdose of sodium pentobarbital (150 mg/kg, ip) and transcardially perfused with $4 \%$ paraformaldehyde solution. The brains were extracted and stored overnight in the same fixative solution and then transferred to $30 \%$ sucrose solution for an additional 72 hours before being cut into $50 \mu \mathrm{m}$ thick coronal sections on a freezing microtome (Leica, Mannheim, Germany). Sections were kept at $-20^{\circ} \mathrm{C}$ in a cryo-protectant solution [30\% glycerol (v/v) and $30 \%$ ethylene glycol $(\mathrm{v} / \mathrm{v})$ in $0.1 \mathrm{M}$ phosphate buffer $(\mathrm{PB}, \mathrm{pH}: 7.4)$ ] until processed for immunohistochemistry. Sections were first incubated for 1 hour in blocking PBS solution containing 4\% donkey serum and $0.3 \%$ Triton X-100 (Sigma, St. Quentin Fallavier, France). They were then incubated for 24 hours at $4^{\circ} \mathrm{C}$ with MW8 antibody (Developmental Studies Hybridoma Bank, The University of lowa, Department of Biological Sciences, lowa City, IA) (16-17) diluted 1:64000 with the 
blocking solution. They were then rinsed in PBS and incubated for 1 hour in blocking solution containing an Alexa568-conjugated donkey anti-mouse antibody (1:500, Jackson Immunoresearch). Sections were washed, mounted on glass slides and covered with a coverslip.

Mosaic images of entire brain sections were acquired with a Leica DM6000B microscope (Leica Microsystems, Mannheim, Germany) equipped with a Qimaging RETIGA EXI camera, using Micromanager to control the acquisition process with a 20X lens (Leica Microsystems, Mannheim, Germany). To calculate the mean number of $\mathrm{mHtt}$ aggregates $/ \mathrm{mm}^{2}$ per region of interest, 6 independent measures (from separate sections and hemispheres) were performed and averaged for each mouse brain. Quantification was conducted by contouring entire areas for each of the following regions of interest. The striatum and motor cortex were analyzed approximately at $0.5 \mathrm{~mm}$ anterior to bregma level and the sensory cortex, CA1, CA2, CA3 and Dentate gyrus of the dorsal hippocampus and amygdala at $2 \mathrm{~mm}$ posterior to bregma. "Find maxima segmentation" (ImageJ software) was applied within the selected area to quantify mHtt aggregates.

An initial experiment of double labeling with both MW8 and DAPI staining demonstrated that aggregates detected by the MW8 antibody were essentially found in the nucleus revealed with DAPI. In addition, aggregates revealed by MW8 staining were essentially in large and bright spots that were selectively identified by "find maxima" while diffuse background was not.

\section{$\underline{\text { Statistics }}$}

Raw or transformed (log or square root) data that have met the requirement for parametric statistical application (normal distribution, equality of variances) were analyzed by Student t-test or ANOVA and data were presented as mean \pm SEM. Otherwise, non-parametric tests (i.e. MannWhitney test) were used and data were summarized as median \pm interquartile and/or min-max range. Statistical significance was set at $p<.05$. Further principal component analysis was performed to inter-relate measures within groups and reduce to a small number of principal components that 
combine different variables that co-vary. Proportions of animals displaying clasping summarized in Fig. 1b, were compared between sexes using $\mathrm{Ch}^{2}$. These analyses were conducted using Statistica, GraphPad Prism or Statview softwares.

\section{Results}

\section{Weight, clasping, inverted grid test, rotarod}

Weights collected at the last day of the experiment indicated that female mice weighed less than male mice $(F(1,58)=69.775, p<.0001$, Fig. 1a), and this sex difference was evident in WT mice $(p<.0001)$ but not $R 6 / 1(\mathrm{Tg})$ mice $(p>.05)$ (genotype $x$ sex interaction; $F(1,58)=25.854, p<.0001) .57 .14$ $\% \pm 11.17$ of male and $46.75 \% \pm 12.81$ of female Tg mice exhibited clasping, while none of the WT mice did so (genotype effect; $\mathrm{Ch}^{2}=17.302, \mathrm{p}<.0001$, sex effect in $\mathrm{R} 6 / 1$ mice: $\mathrm{Ch}^{2}=.383$, n.s. Fig. 1 b). The latency to fall from the grid was not significantly different between the 2 genotypes (MannWhitney $U=U^{\prime}=75$, n.s.), and female mice, irrespective of genotype, tended to display overall longer latencies than males $\left(U=105.5, U^{\prime}=230.5, p=.055\right.$, Fig. 1c).

Across 3 trials, no significant differences between genotypes and sexes, nor interaction between the two factors, was found for latencies to fall from the rotarod. When scores for the $3^{\text {rd }}$ trial only were considered, female mice, regardless of genotype, outperformed male mice $(F 1,58)=4.244, p=.044$, Fig. $1 d)$. 
a

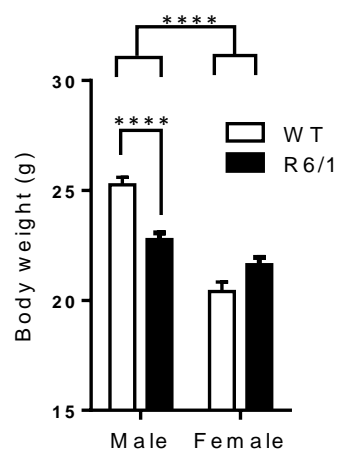

b

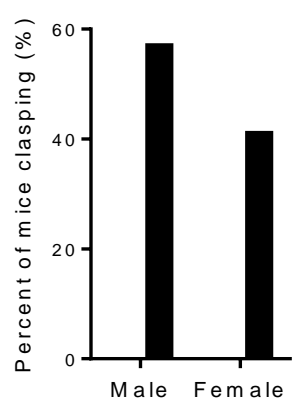

C

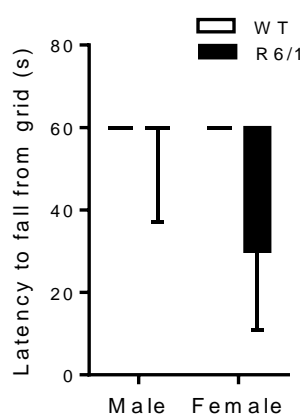

d

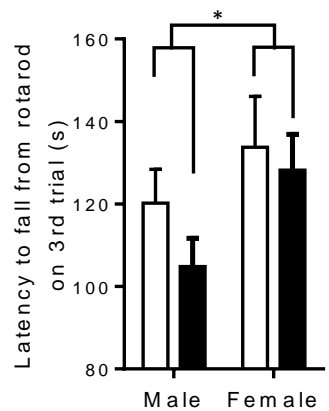

Fig. 1. Body weight (a), percentage of mice displaying clasping (b), latency to fall from the grid (c) and latency to fall from the rotarod on $3^{\text {rd }}$ trial (d) in male and female R6/1 and WT mice at 3 months. In b, none of WT mice displayed clasping. In c, note that all WT mice displayed maximal latency (60s) to fall from the grid. Data are presented as mean $\pm \operatorname{SEM}(a, d)$ and median \pm interquartile and min-max (whiskers) ranges (c). ${ }^{*} \mathrm{p}<.05, * * * *, \mathrm{p}<.0001$.

\section{Elevated plus maze, social and nest tests}

Tg mice entered open arms more often than WT mice $(F(1,57)=20.95, p<.0001)$ and male mice visited open arms more often than female mice $(F(1,57)=7.95, p=0.007)$. However, there existed no interaction between genotype and $\operatorname{sex}(F(1,57)<1$, n.s.) (Fig. 2a). However, an effect of genotype was found when the analysis was performed on percent time spent in open arms $(F(1,57)=10.42, p=.002)$ (Fig. 2b). Therefore, Tg mice, in general, can be said to be less anxious than WT littermates since they visited and spent more time in open arms of the plus maze.

In general, Tg mice constructed nests of significantly poorer quality than WT littermates (Mann-Whitney, $\left.\mathrm{U}=27, \mathrm{U}^{\prime}=885, \mathrm{p}<.0001\right)$, this in both sexes $(\mathrm{p}<.0001$ in males, $\mathrm{p}<.05$ in females, Fig. 2c). $\operatorname{Tg}$ mice, irrespective of sex, spent less time engaging in social behavior $(F(1,58)=8.083, p=.0062$,

Fig. $2 d)$, significant genotype associated difference was found in male mice $(p<.01)$. On the contrary, 
R6/1 mice spent overall more time engaging in non-social behavior $(F(1,58)=9.49, p=.003$, Fig. 2e), with significant difference in males $(p<.0001)$. No significant difference was found for aggressive behaviors, even though male mice in general tended to spend more time engaging in aggressive behavior than females ( $U=124, U^{\prime}=191, p=.074$, Fig. $\left.2 f\right)$.
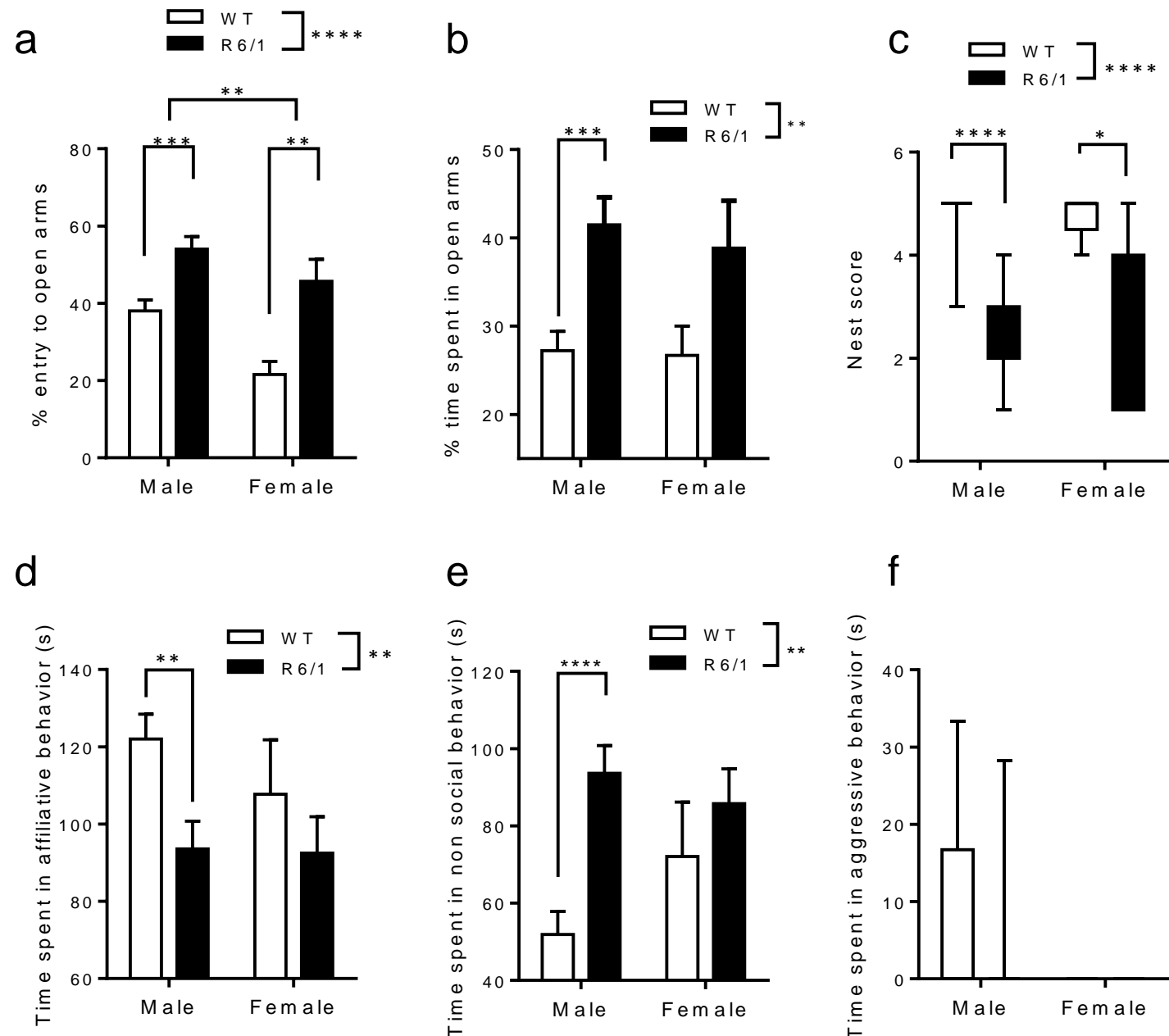

e

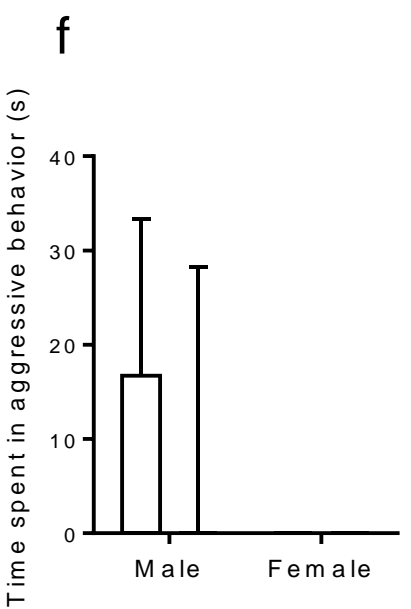

Figure 2. Behavioral performances in elevated plus-maze (a-b), nest test (c) and social interaction test (d-f) in 3 month-old R6/1 and WT mice. Percent entries to open arms (a) and percent time spent in open arms in the plus maze (b) as well as engaging in social (d), non-social (e) and aggressive (f) behaviors during the $3 \mathrm{~min}$ social interaction with a congener. Data are presented as mean \pm SEM $(a, b, d, e)$ and median \pm interquartile and min-max (whiskers) ranges $(c, f) .{ }^{*}$ Significant effect at $p<.01$, $* * \mathrm{p}<.01, * * * \mathrm{p}<.001, * * * * \mathrm{p}<.0001$. 


\section{Short-term spatial memory in the Y-maze, and acquisition and long-term retention in the Barnes}

$\underline{\text { maze }}$

During the 2 min test trial in the Y-maze, Tg mice travelled significantly less distance in the maze than WT mice $(F(1,58)=25.563, p<.0001)$, but no sex difference, or interaction, was observed for this measure (Fig.3a). No significant differences in spatial recognition memory index were found between genotypes $(F(1,58)=2.745$, n.s. $)$, or $\operatorname{sex}(F(1,58)<1$, n.s. Fig. $3 b)$, even though male Tg mice had a tendency to perform poorer than male WT mice (Fig. 3b). All groups of mice performed significantly above chance level (33.3\%) (one-sample t-test, $p<.05)$.

a

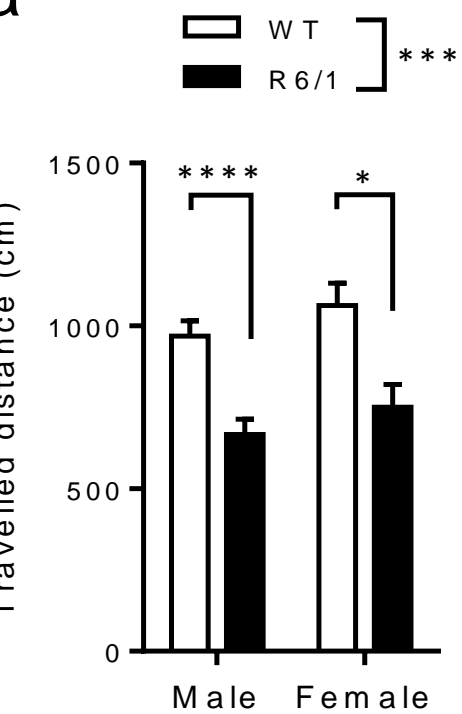

b

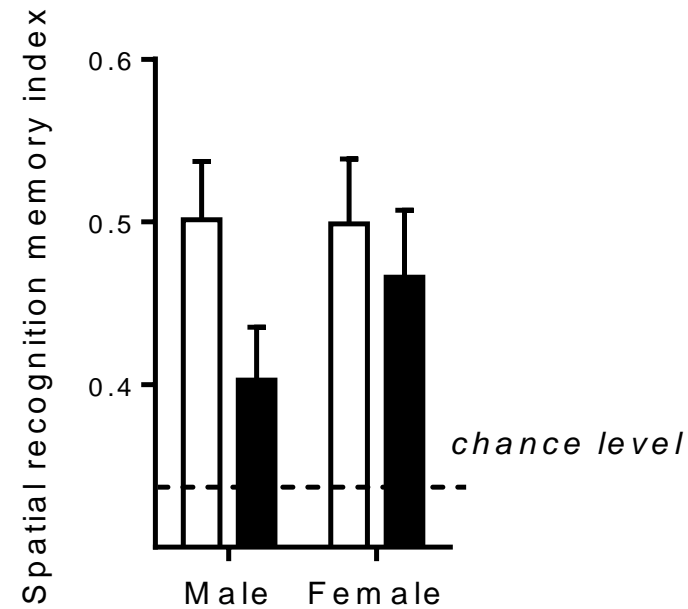

Fig. 3. Short-term spatial recognition memory in the Y-maze. Travelled distance (in $\mathrm{cm}$ ) as well as spatial recognition memory index (with 33.33\% chance level) assessed during the 2-min test phase in 3 month-old R6/1 and WT mice. Data are presented as mean \pm SEM. ${ }^{*} p<.05, * * * *, p<.0001$.

Throughout the Barnes maze acquisition trials, Tg mice, irrespective of sex, required longer latencies to reach the safe hole $(F(1,58)=41.102, p<.0001)$; genotype associated differences were significant for all 6 trials ( $p<.05$ for all trials) (Fig. 4a). Nevertheless, Tg mice did demonstrate 
learning, as latencies decreased significantly over the trials (trial effect in $\operatorname{Tg}$ mice: $F(5,290)=24.138$, $p<.0001)$. No sex difference was found during the acquisition $(F(1,58)<1$, n.s.). The latency measure might be influenced by the decreased locomotion previously found in the Y-maze in Tg mice. We therefore performed analyses of the speed of movement and distances travelled to reach the safe hole in the maze. As expected, R6/1 mice of both sexes displayed lower speed throughout the 6 trials $(F(1,58)=24.912, p<.0001)$; significant differences between the genotypes were found on each trial $(p<.05)$ except for the $5^{\text {th }}$ trial (Fig. 4b). However, all mice were able to improve their speed with training (trial effect: $F(5,290)=13.42, p<.0001$ ), the increase of the speed was even more evident in R6/1 mice than WT mice (genotype $x$ trial interaction: $F(5,290)=3.049, p=.011$ ). While higher latencies may be due to lower locomotion speed in R6/1 mice, they still travelled a significantly longer distance through the 6 trials $(F(1,58)=18.175, p<.0001)$, indicating their protracted learning (Fig. $4 c)$. Independent of sex, $\operatorname{Tg}$ mice travelled significantly longer distances during the $2^{\text {nd }}, 4^{\text {th }}$ and $6^{\text {th }}$ trials $(p<.05)$ 

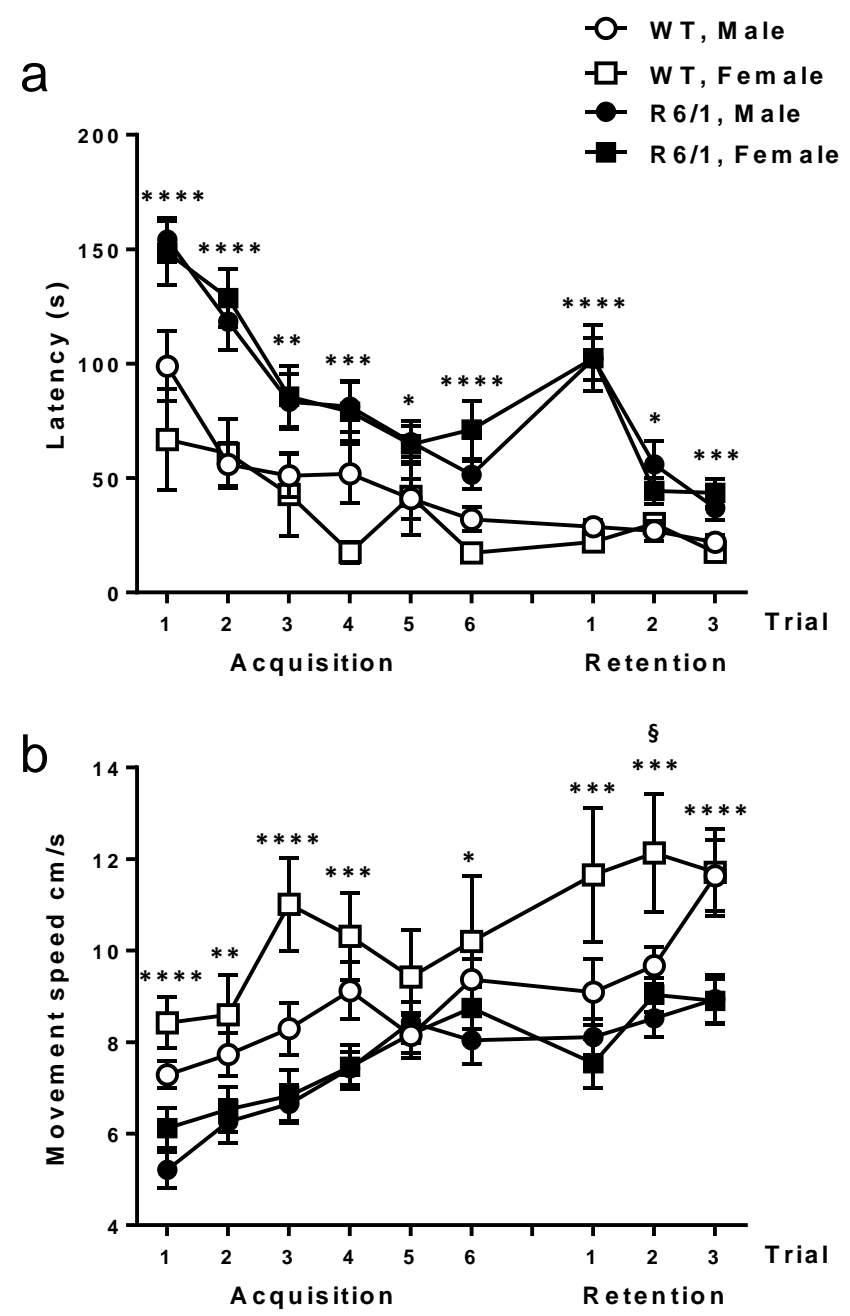

C

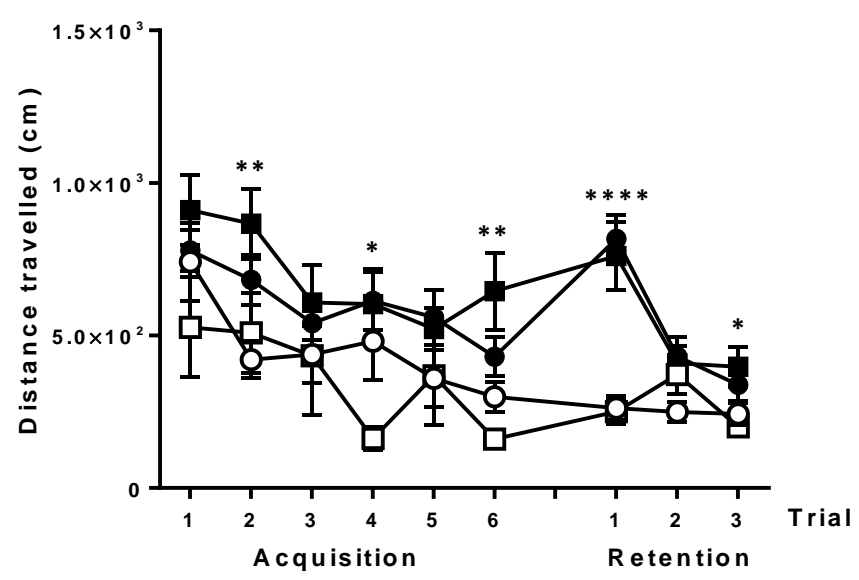

Fig. 4. Latencies in second (a) and travelled distance in $\mathrm{cm}$ (c) to reach the safe hole as well as speed of the locomotion (b) in the Barnes maze during acquisition ( 6 trials) and retention ( 3 trials) testing in 3 month-old R6/1 and WT mice. * Significant genotype effect at $p<.05, * *<.01, * * * p<.001, * * * *$ $p<.0001, \S$ significant sex effect at $p<.05$. Data ware presented as mean \pm SEM. 
During the 3 retention trials that were performed 24 hours following the acquisition, $\mathrm{Tg}$ mice had longer latencies $(F(1,58)=44.58, p<.0001$, Fig. 4a), displayed lower moving speed $(F(1,58)=25.702$, $\mathrm{p}<.0001$, Fig. 4b) and travelled longer distances $(F(1,58)=39.085, p<.0001$, Fig. 4c). Interestingly, main effects of genotype seen for latency and distance measures were due to the high scores of $\mathrm{Tg}$ mice on the first retention trial [genotype $x$ trial interaction for latency $(F(2,116)=18.019, p<.0001)$ and distance $(F(2,116)=11.423, p<.0001)]$, while such an effect was not found for speed $(F(2,116)<1$, n.s.). This indicates that deteriorated performances in R6/1 mice (seen in latency and distance) are specific to mnemonic domain, but not purely locomotion impairments.

To assess the degree to which mice displayed improvement between the acquisition and retention trials, repeated measures 2-way ANOVA was performed on latency and distance on the last acquisition and first retention trials. This analysis indicated that, while WT mice improved over the 2 trials by decreasing latency and distance, $\mathrm{Tg}$ mice rather displayed an increase of latency $(F(1,58)=55.901, p<.0001)$ and distance $(F(1,58)=3.103, p=.083)$, revealing a deterioration in performances, suggesting their forgetting of the information acquired 24 hours earlier.

During retention trials, the position of the cued safe hole was displaced to another location in the maze. Frequency distribution of spatial and cue responses demonstrated that $62.5 \%$ of WT mice and $54.1 \%$ of $\mathrm{Tg}$ mice chose the previous spatial location; these proportions were not significantly different between genotypes in all 3 trials $\left(\mathrm{Chi}^{2}, \mathrm{z}=2.848\right.$, n.s.). However, the number of spatial responses was significantly higher than that of cue responses across 3 trials in WT (1.88 \pm 0.21 versus $1.12 \pm 0.21 ; p=0.0156)$ but not $\operatorname{Tg}$ mice $(1.55 \pm 0.16$ versus $1.32 \pm 0.21, p>.05)$ that showed the same prevalence of the spatial versus cue responses in Tg mice. Finally, the number of errors (visits of unsafe holes) was markedly higher in Tg than WT mice, and this irrespective of the sex and trials (Genotype effect: $F(1,58)=27.945 ; p<0.0001)$. Therefore, the use of the spatial strategy was attenuated in R6/1 mice and was not compensated by an increased use of the cued strategy, 
indicating impairments in both hippocampus-dependent and striatum-dependent learning and memory processing.

\section{Correlation matrix and principal component analysis of behavior}

Correlation matrix was calculated to interrelate behavioral measures (and $\mathrm{mHtt}$ inclusions). As could be seen in Table 1, latency to fall from rotarod was negatively correlated with body weight, and positively correlated with spatial recognition memory index in the $\mathrm{Y}$ maze. Time spent in open arms in the elevated plus maze was positively correlated with the presence of clasping and distance travelled to reach goal in the Barnes maze; the less anxious, the more clasping and the longer distance to reach the shelter in the Barnes maze. Finally, distance to find the safe hole on the last trial in the Barnes maze was negatively correlated with body weight and long-term memory loss, and positively correlated with time spent in open arms in the elevated plus maze.

Table 1. Correlation matrix involving 12 behavioral scores (in black) and $\mathrm{mHtt}$ inclusions in 8 different neuroanatomical regions (in blue). Values in bold indicate statistically significant coefficients of correlation. Rotarod: latency (sec) to fall from rotarod on $3^{\text {rd }}$ trial, Anxiety: \% time spent in open arms, Grip: latency (sec) to fall from the grid, Social, aggressive: time (sec) spent in social and aggressive behaviors during social interaction with a female NMRI stimulus mouse, Mobility: distance travelled $(\mathrm{m})$ during Y-maze testing, Y-maze: spatial recognition memory index, LTM loss: difference of latency ( $s$ ) to find the safe hole between the last trial of acquisition and the first trial of retention , Acquisition: distance $(\mathrm{cm})$ to find a safe hole in the Barnes maze on $6^{\text {th }}$ trial of acquisition.

\section{Quantification $\mathrm{mHtt}$ in different neural regions in $\mathrm{R} 6 / 1$ mice}

We used the MW8 antibody that stains frank intranuclear inclusions (16-17), this specificity was confirmed by co-labeling with DAPI staining (not shown). The number of $\mathrm{mHtt}$ inclusions in R6/1 mouse brains were counted and expressed by $\mathrm{mm}^{2}$ of surface in each region of interest (Fig. 5a-c). One mouse from each sex was discarded from the analysis due to a technical problem during immunocytochemical processing. We found no differences in levels of $\mathrm{mHtt}$ inclusions associated 
with sex in all regions of interest (Fig. 5d). However, different regions possessed different numbers of $\mathrm{mHtt}$ inclusions. For example, the number was higher in any subregion of the hippocampus than other regions (Wilcoxon rank test for data combined from both sexes, $\mathrm{p}<.0001$ for all comparisons, Fig. 5d), DG and CA1 were the highest and CA2 and CA3 the lowest within the hippocampus. The inclusion number was higher in the striatum and sensory cortex than motor cortex amygdala (Wilcoxon rank test all $p<.0001$, Fig. $5 d$ ).
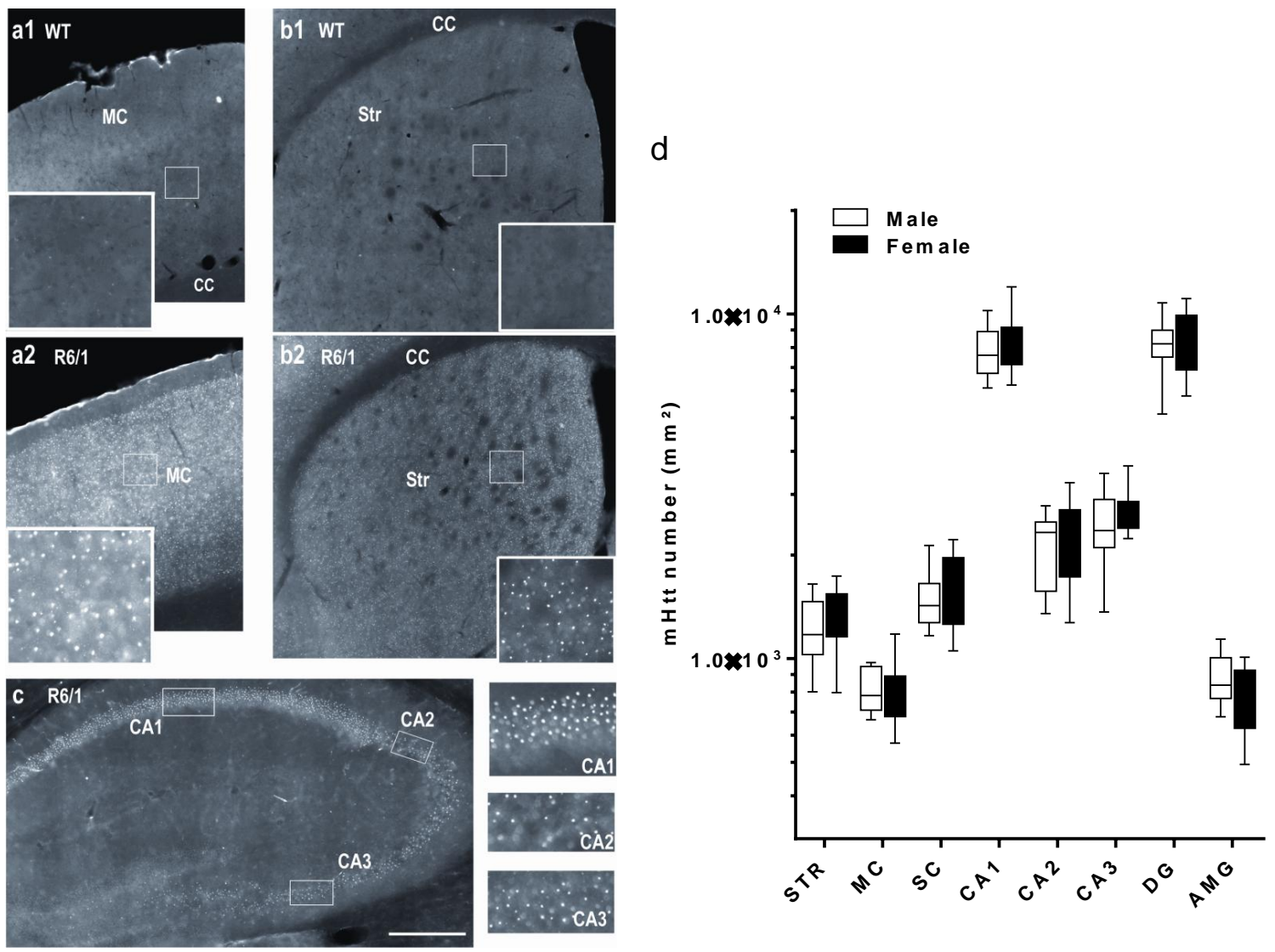

Fig.5. Mutant Huntingtin (mHtt) labeling in 3 month-old male $(n=20)$ and female $(n=16) R 6 / 1$ mice. Fluorescent labelling for $\mathrm{mHtt}$ in coronal sections through motor Cortex (MC, a1,a2), striatum (Str, b1,b2), CA1, CA2, and CA3 of the hippocampus (c) in WT and R6/1 mice. White frames detailed in insets. While mHtt aggregates are not detected in WT mice (a1, b1), labelling is clearly detected in R6/1 mice $(a 2, b 2, c)$. Scale bar: $500 \mu \mathrm{m}$. mHtt numbers per $\mathrm{mm}^{2}$ in different regions (d). Data in (d) are presented as median \pm interquartile and min-max (whiskers) ranges. STR, striatum; MC, motor cortex; SC, sensory cortex; DG, dentate gyrus; CA1, hippocampal CA1 subfield; CA2, hippocampal CA2 subfield; CA3, hippocampal CA3 subfield; AMG, amygdala. 


\section{Correlation matrix for behavioral/physiological and $\mathrm{mHtt}$ data in $\mathrm{R6} / 1$ mice}

Correlation matrix (Table 1) revealed that the number of $\mathrm{mHtt}$ inclusions in the striatum was significantly correlated with that of the motor and sensory cortex, CA1, CA2 and dentate gyrus (DG) of the hippocampus (all $p<.05)$, but not with that of amygdala and CA3 ( $p>.05)$. The motor cortex inclusions were correlated with the CA1 and DG of the hippocampus and the sensory cortex $(p<.05)$ ), but not the CA2, CA3, and amygdala $\mathrm{mHtt}(\mathrm{p}>.05)$.

In addition, the number of aggregates in the striatum was negatively correlated with grip strength $(p=-.376, p=.026)$, while that of the sensory cortex was positively correlated with $\%$ time spent in open arms in the plus-maze $(p=.424, p=.011)$ as well as distance travelled to reach the safe hole at the end of the Barnes maze acquisition $(p=.404, p=.016)$. This analysis also revealed that the $\mathrm{mHtt}$ inclusions in the CA2 of the hippocampus was positively correlated with travelled distance in the Y-maze $(p=.380, p=.024)$.

\section{Principal component analysis}

Principal component analysis was performed to reduce behavioral and pathophysiological variables to a few principal components that are related to one another. This analysis on R6/1 mice of both sexes extracted 8 principal components (explaining $78.8 \%$ of the total variance) with Eigenvalue greater than 1 . We considered a correlation above 0.40 important for interpretation. The first principal component, explaining $21.9 \%$ of the variance, was correlated with the level of $\mathrm{mHtt}$ inclusions in all regions of interest except for the amygdala (Fig. 6, X-axis). This component also increased with \% time spent in open arms of the elevated plus maze and decreased with latency to fall from the grid. Therefore, the high level of $\mathrm{mHtt}$ in most regions was associated with hypo-anxiety and decreased muscle strength in Tg mice. 
The second principal component, explaining $12.8 \%$ of the variance (Fig. 6, Y-axis) increased with amygdala and to a less extent with CA2 $\mathrm{mHtt}$, body weight and long-term memory loss in the Barnes maze. The same component also decreased with the latency to fall from rotarod, time spent in open arms in the elevate plus maze, the presence of clasping, spatial recognition memory index in the Y-maze, and distance travelled to the shelter in the Barnes maze. Therefore, high numbers of inclusion in the amygdala and CA2 of the hippocampus were associated with reduced short- and long-term memory and motor coordination abilities on one hand, and with less HD associated phenotypic changes such as body weight loss, clasping, hypo-anxiety, etc., on the other hand. The $4^{\text {th }}$ component ( $8.4 \%$ of the total variance) increased with motor cortex inclusions, aggressiveness and body weight, and decreased with mobility in the $Y$ maze. The remaining components, each explaining less than $10 \%$ of the total variances did not correlate with $\mathrm{mHtt}$ inclusions.

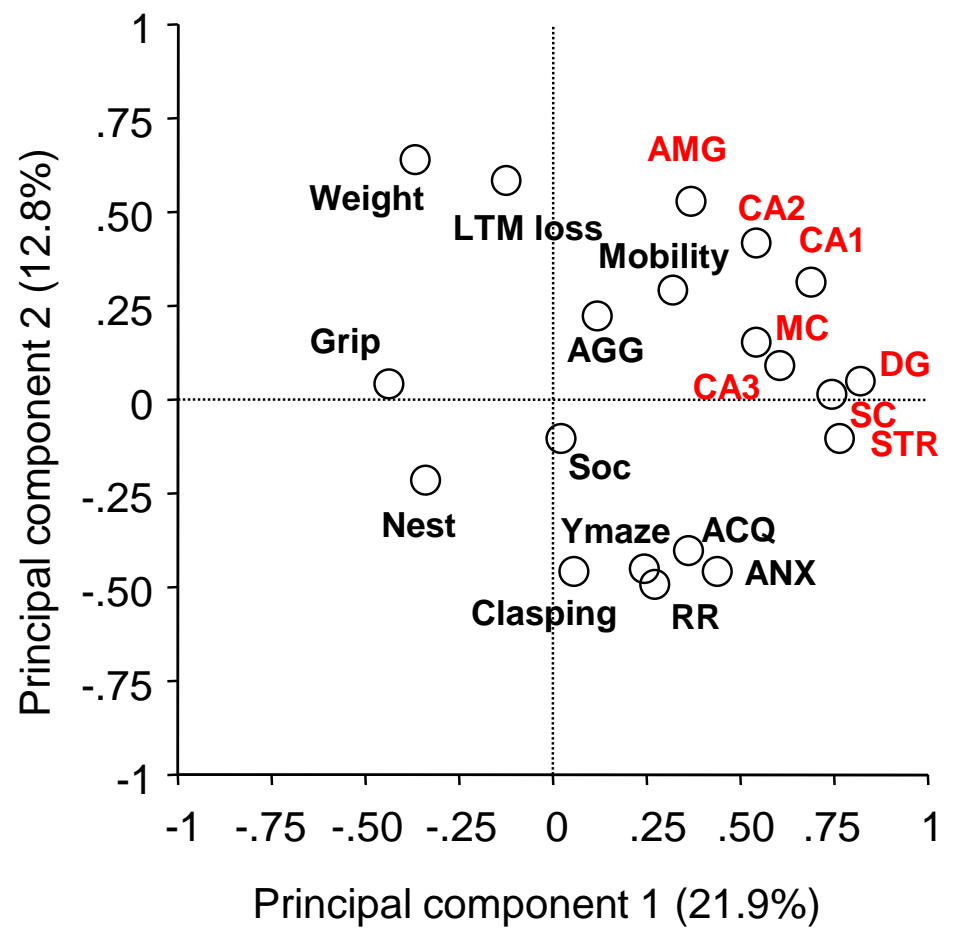

Fig. 6. Principal component analysis involving 12 behavioral variables (in black) and $\mathrm{mHtt}$ levels in 8 neuroanatomical regions (in red) in both male $(n=19)$ and female $(n=16) R 6 / 1$ mice of 3 months of age. Score plots of variables were presented over the $1^{\text {st }}$ and $2^{\text {nd }}$ components. Abbreviations: ACQ: distance to the safe hole on the last $6^{\text {th }}$ trial during acquisition in the Barnes maze, LTM loss: longterm memory loss (increase of latency to reach the goal between the last trial of acquisition and the 
first trial of retention test) in Barnes maze, AMG: amygdala, MC: motor cortex, Grip: grip strength, Mobility: distance travelled in the Y-maze, SC: sensory cortex, Soc: social behavior, ANX: \% time spent in open arms in the elevated plus maze, STR: striatum, Y-maze: spatial recognition memory index in the Y-maze, RR: latency to fall from rotarod, Agg: aggressivity

\section{Discussion}

The current study aimed at establishing correlations between behavioral/physiological deficits and the number of $\mathrm{mHtt}$ aggregates in different regions of interest in R6/1 HD transgenic mice. R6/1 mice were studied at 3 months of age, as this age corresponds to a turning point where some animals develop behavioral and physiological changes, while others remain relatively asymptomatic. This variability may be explained by the potential underlying variability in histopathological severity. The sex-associated difference may also contribute to the inter-individual variance within Tg mice. It is of note that mouse models display low levels of neurodegeneration, that is detected only long after the onset of overt symptoms (ref PMID: 10869421). At an earlier age no evidence for neurodegeneration is found in the brain (Mangiarini et al., 1996; PMID: 10869421, Pietropaolo et al.,2015)

We confirmed that R6/1 mice are deficient in instinctive, locomotor, anxiety related behaviors as well as acquisition and long-term retention memory in the Barnes maze. Significant differences were also found between the sexes; as compared to male counterparts, female R6/1 mice displayed no or less severe changes in weight loss, hypo-anxiety, social, non-social and nest construction abilities. Remarkable deficits were noticed in the capacity of $\mathrm{Tg}$ mice to retain the location of the safe hole in the open-field Barnes maze overnight, while short-term memory in the $Y$ maze remained relatively unaltered at this age. This may suggest that long-term memory is altered earlier in the disease progression than short-term memory. Moreover, we cannot exclude a potential effect of the hypo-anxious phenotype of $\mathrm{Tg}$ mice on the acquisition and retention performances in the Barnes maze, that rely on aversive reinforcement. On the other hand, changes in anxiety levels 
may have less influence on the short-term memory assessed in the closed Y-maze. In addition, the analysis of spatial versus cue strategy used by mice when choosing the shelter position during the retention test in the Barnes maze demonstrated somewhat different behaviors. A greater proportion of mice chose according to extramaze against local cue in WT mice, while this preference was not evident in Tg mice. Furthermore, R6/1 mice displayed increased visits of holes that are associated with neither spatial nor local cue, demonstrating deteriorated capacity for retention over a $24 \mathrm{hr}$ delay. Our behavioral data confirm previous observations of our group (18-20) as well as other reports (21-23).

We also found and confirmed the presence of $\mathrm{mHtt}$ aggregates at 3 months of age in different anatomical regions. The number of $\mathrm{mHtt}$ aggregates in $\mathrm{R} 6 / 1$ mice, while not differing between the two sexes, differed between the examined regions. Our study also demonstrated that sex differences exist in the expression of physiological and behavioral phenotypes, but not the $\mathrm{mHtt}$ levels in brain regions investigated here. Due to the high density of cellular organization, different subfields of the hippocampus contained a higher number of $\mathrm{mHtt}$ per unit of area than other neuroanatomical regions. Significant correlations among pairs of regions were also found, albeit that only subtle differences in the strength of correlations exist. However, it could also be noticed that the number of aggregates in amygdala remained uncorrelated, and that aggregates in the CA2 and CA3 subfields of the hippocampus are less correlated with inclusions expressed in other regions. This observation is informative of the fact that the spatial/neuroanatomical and temporal progression of the $\mathrm{mHtt}$ is heterogeneous, and certain brain areas do not follow the same $\mathrm{mHtt}$ accumulation pattern displayed by other regions investigated here. Our results demonstrate that the increased number of $\mathrm{mHtt}$ aggregates in the striatum is related to a decrease in grip strength, and the augmented $\mathrm{mHtt}$ in the sensory cortex is related to a hypo-anxious phenotype. Interestingly, the number of $\mathrm{mHtt}$ in $\mathrm{CA} 2$ of the hippocampus is also correlated with travelled distance in the $\mathrm{Y}$-maze test mimicking WT mouse profile: this observation suggests the beneficial impact of $\mathrm{mHtt}$ on locomotor ability. 
Advanced principal component analysis revealed that the $\mathrm{mHtt}$ levels in most regions were correlated with known HD phenotypes, such as deficits in muscle strength and hypo-anxiety, supporting the hypothesized toxic effect of $\mathrm{mHtt}$ inclusions $(4,5)$. This analysis also revealed that the amygdala and to a less extent CA2 aggregates not only correlated with HD phenotypes, i.e. deficits in motor coordination and short- and long-term memory, but also demonstrated a negative relationship with other HD associated changes, such as body mass loss, clasping, hypo-anxiety and acquisition deficits in the Barnes maze. This may suggest a beneficial contribution of aggregates in the amygdala and CA2 field to attenuate behavioral deficits in R6/1 mice, confirming some previous literature suggesting a protective role of $\mathrm{mHtt}(7,24)$.

Our results are not congruent with those obtained by Rattray and colleagues who demonstrated no clear relationship between the brain physiopathology and the severity of behavioral impairments in $\mathrm{R} 6 / 2$ and $\mathrm{R} 6 / 1$ mice $(25,26)$ as well as HdhQ150 mice $(27)$. This may be due to the use of longitudinal plan in their study in which biochemical and physiopathological measures collected at the end stage of the disease could not be properly correlated with different behaviors assessed at different ages and time points. Our study was designed to overcome this issue by promptly collecting both types of data in a large cohort of R6/1 mice of the same age. However, our study is partially congruent with Graham et al, who demonstrated that the onset and progression of HD were correlated with the levels of $\mathrm{mHtt}$ in the YAC128 mouse model of HD

As mentioned above, the sex-related differences in behavior were not accompanied by those in $\mathrm{mHtt}$ levels in the brain. While several studies demonstrated differences between sexes, their $\mathrm{mHtt}$ loads remain indistinguishable (e.g. 26), such comparison of $\mathrm{mHtt}$ levels remained seldom. While slightly lower sample size in female mice (9 WT and 17 TG) might have contributed to the statistical insignificant difference in $\mathrm{mHtt}$ levels between sexes, our data suggest that behavioral changes are not entirely and solely dependent on the $\mathrm{mHtt}$ pathology reported here. Therefore, 
physiology unique to each sex should be considered as a whole to explain behavioral changes in mouse models of HD.

\section{Conclusion}

Our study demonstrates that the presence of a large number of mHtt inclusions may be harmful and have a functional impact on the neural integrity of many regions of the limbic circuit involved in cognitive and motor behaviors. However, we found that their harmful effects were dependent on the neuroanatomical regions considered, which raises questions about the specific roles played by $\mathrm{mHtt}$ in different cell populations and neural networks. Investigating the specificity of $\mathrm{mHtt}$ with respect to different cell populations may be important in order to obtain a better understanding of the $\mathrm{Htt}$ physiopathology in HD.

\section{Acknowledgement}

Authors wish to thank Gilles Courtand for help with Image j software, and Laurence Decortes for brain slice processing. This work was supported by the Hereditary disease foundation, New York.

\section{Conflict of interest}

The authors have no conflict of interest to report

\section{References}


1. The Huntington's Disease Collaborative Research Group. A novel gene containing a trinucleotide repeat that is expanded and unstable on Huntington's disease chromosomes. Cell. 1993;72(6):971-83.

2. Davies SW, Turmaine M, Cozens BA, DiFiglia M, Sharp AH, Ross CA, et al. Formation of neuronal intranuclear inclusions underlies the neurological dysfunction in mice transgenic for the HD mutation. Cell. 1997;90(3):537-48.

3. Difiglia M, Sapp E, Chase KO, Davies SW, Bates GP, Vonsattel JP, et al. Aggregation of huntingtin in neuronal intranuclear inclusions and dystrophic neurites in brain. Science. 1997;277(5334):1990-3.

4. Chopra V, Fox JH, Lieberman G, Dorsey K, Matson W, Waldmeier P, et al. A small-molecule therapeutic lead for Huntington's disease: preclinical pharmacology and efficacy of C2-8 in the R6/2 transgenic mouse. Proc Natl Acad Sci U S A. 2007;104(42):16685-9.

5. Zhang X, Smith DL, Meriin AB, Engemann S, Russel DE, Roark M, et al. A potent small molecule inhibits polyglutamine aggregation in Huntington's disease neurons and suppresses neurodegeneration in vivo. Proc Natl Acad Sci U S A. 2005;102(3):892-7.

6. Slow EJ, Graham RK, Osmand AP, Devon RS, Lu G, Deng Y, et al. Absence of behavioral abnormalities and neurodegeneration in vivo despite widespread neuronal huntingtin inclusions. Proc Natl Acad Sci U S A. 2005;102(32):11402-7.

7. Miller J, Arrasate M, Shaby BA, Mitra S, Masliah E, Finkbeiner S. Quantitative relationships between huntingtin levels, polyglutamine length, inclusion body formation, and neuronal death provide novel insight into huntington's disease molecular pathogenesis. J Neurosci. 2010;30(31):10541-50.

8. Arrasate M, Mitra S, Schweitzer ES, Segal MR, Finkbeiner S. Inclusion body formation reduces levels of mutant huntingtin and the risk of neuronal death. Nature. 2004;431(7010):805-10.

9. Bodner RA, Outeiro TF, Altmann S, Maxwell MM, Cho SH, Hyman BT, et al. Pharmacological promotion of inclusion formation: a therapeutic approach for Huntington's and Parkinson's diseases. Proc Natl Acad Sci U S A. 2006;103(11):4246-51.

10. Saudou F, Finkbeiner S, Devys D, Greenberg ME. Huntingtin acts in the nucleus to induce apoptosis but death does not correlate with the formation of intranuclear inclusions. Cell. 1998;95(1):55-66.

11. Deacon RM. Assessing nest building in mice. Nat Protoc. 2006;1(3):1117-9.

12. Martel G, Blanchard J, Mons N, Gastambide F, Micheau J, Guillou JL. Dynamic interplays between memory systems depend on practice: the hippocampus is not always the first to provide solution. Neuroscience. 2007;150(4):743-53.

13. Ciamei A, Morton AJ. Progressive imbalance in the interaction between spatial and procedural memory systems in the R6/2 mouse model of Huntington's disease. Neurobiol Learn Mem. 2009;92(3):417-28.

14. Baudonnat M, Guillou JL, Husson M, Bohbot VD, Schwabe L, David V. Morphine Reward Promotes Cue-Sensitive Learning: Implication of Dorsal Striatal CREB Activity. Front Psychiatry. 2017;8:87.

15. Schwabe L, Dalm S, Schachinger H, Oitzl MS. Chronic stress modulates the use of spatial and stimulus-response learning strategies in mice and man. Neurobiol Learn Mem. 2008;90(3):495-503.

16. Ko J, Ou S, Patterson PH. New anti-huntingtin monoclonal antibodies: implications for huntingtin conformation and its binding proteins. Brain Res Bull. 2001;56:319-329.

17. Bayram-Weston Z, Jones L, Dunnett SB, Brooks SP. Comparison of mHTT antibodies in Huntington's Disease mouse models reveal specific binding profiles and steady-state ubiquitin levels with disease development.PLoS One. 2016;11(5):e0155834.

18. Pietropaolo S, Delage P, Cayzac S, Crusio WE, Cho YH. Sex-dependent changes in social behaviors in motor pre-symptomatic R6/1 mice. PLoS One. 2011;6(5):e19965.

19. Lebreton F, Cayzac S, Pietropaolo S, Jeantet Y, Cho YH. Sleep Physiology Alterations Precede Plethoric Phenotypic Changes in R6/1 Huntington's Disease Mice. PLoS One. 2015;10(5):e0126972. 
20. Ragot A, Pietropaolo S, Vincent J, Delage P, Zhang H, Allinquant B, et al. Genetic deletion of the Histone Deacetylase 6 exacerbates selected behavioral deficits in the R6/1 mouse model for Huntington's disease. Brain and behavior. 2015;5(9):e00361.

21. Naver B, Stub C, Moller M, Fenger K, Hansen AK, Hasholt L, et al. Molecular and behavioral analysis of the R6/1 Huntington's disease transgenic mouse. Neuroscience. 2003;122(4):1049-57.

22. Nithianantharajah J, Barkus C, Murphy M, Hannan AJ. Gene-environment interactions modulating cognitive function and molecular correlates of synaptic plasticity in Huntington's disease transgenic mice. Neurobiol Dis. 2008;29(3):490-504.

23. Brooks SP, Janghra N, Workman VL, Bayram-Weston Z, Jones L, Dunnett SB. Longitudinal analysis of the behavioural phenotype in R6/1 (C57BL/6J) Huntington's disease transgenic mice. Brain Research Bulletin. 2012;88(2-3):94-103.

24. Chen JY, Parekh M, Seliman H, Bakshinskaya D, Dai W, Kwan K, et al. Heat shock promotes inclusion body formation of mutant huntingtin $(\mathrm{mHtt})$ and alleviates $\mathrm{mHtt}$-induced transcription factor dysfunction. J Biol Chem. 2018;293(40):15581-93.

25. Rattray I, Smith E, Gale R, Matsumoto K, Bates GP, Modo M. Correlations of behavioral deficits with brain pathology assessed through longitudinal MRI and histopathology in the R6/2 mouse model of HD. PLoS One. 2013;8(4):e60012.

26. Rattray I, Smith EJ, Crum WR, Walker TA, Gale R, Bates GP, et al. Correlations of behavioral deficits with brain pathology assessed through longitudinal MRI and histopathology in the R6/1 mouse model of Huntington's disease. PLoS One. 2013;8(12):e84726.

27. Rattray I, Smith EJ, Crum WR, Walker TA, Gale R, Bates GP, et al. Correlations of behavioral deficits with brain pathology assessed through longitudinal MRI and histopathology in the HdhQ150/150 mouse model of Huntington's disease. PLoS One. 2017;12(1):e0168556.

28. Graham RK, Slow EJ, Deng Y, Bissada N, Lu G, Pearson J, et al. Levels of mutant huntingtin influence the phenotypic severity of Huntington disease in YAC128 mouse models. Neurobiol Dis. 2006;21(2):444-55. 